\title{
Effectiveness of a thoracic multidisciplinary clinic in the treatment of stage III non-small-cell lung
} cancer

\author{
Eliot L Friedman' \\ Robert J Kruklitis ${ }^{2}$ \\ Brian J Patson' \\ Dennis M Sopka ${ }^{3}$ \\ Michael J Weiss ${ }^{4}$ \\ 'Division of Hematology-Medical \\ Oncology, ${ }^{2}$ Division of Pulmonary and \\ Critical Care Medicine, ${ }^{3}$ Department \\ of Radiation Oncology, ${ }^{4} \mathrm{Health}$ \\ Systems Research and Innovation, \\ Lehigh Valley Health Network, \\ Allentown, PA, USA
}

Video abstract

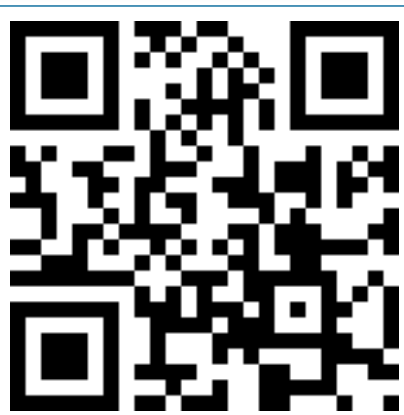

Point your SmartPhone at the code above. If you have a QR code reader the video abstract will appear. Or use: http://youtu.be/KxDxLRfDxM4
Correspondence: Eliot L Friedman Division of Hematology-Medical Oncology, Lehigh Valley Health Network, 1240 South Cedar Crest Boulevard, Suite $4 \mathrm{II}$, Allentown, PA 18103, USA

$\mathrm{Tel}+\mathrm{I} 6104020512$

$\mathrm{Fax}+\mathrm{I} 6104020504$

Email eliot_l.friedman@lvhn.org
This article was published in the following Dove Press journal:

Journal of Multidisciplinary Healthcare

13 June 2016

Number of times this article has been viewed

Introduction: The Institute of Medicine, the American Society of Clinical Oncology, and the European Society of Medical Oncology promote a multidisciplinary approach for the treatment of cancer. Stage III non-small-cell lung cancer (NSCLC) represents a heterogeneous group of diseases necessitating coordination of care among medical, radiation, and surgical oncology. The optimal care of stage III NSCLC underscores the need for a multidisciplinary approach.

Methods: From tumor registry data, we identified all cases of stage III NSCLC seen at Lehigh Valley Health Network between March 2010 and March 2013. The care received by patients when seen in the thoracic multidisciplinary clinic (MDC) was compared with the care received when not seen in the thoracic MDC.

Results: All patients seen in the MDC, compared to $<50 \%$ of patients seen outside the MDC, were evaluated by more than one physician prior to beginning the treatment. Time to initiate treatment was shorter in MDC patients than in non-MDC patients. Patients seen in the MDC had a greater concordance with clinical pathways. A greater percentage of patients seen in the thoracic MDC had pathologic staging of their mediastinum. Patients seen in the MDC were more likely to receive all of their care at Lehigh Valley Health Network.

Conclusion: Multidisciplinary care is essential in the treatment of patients with stage III NSCLC. Greater utilization of MDCs for this complex group of patients will result in more efficient coordination of care, pretreatment evaluation, and therapy, which in turn should translate to improve patients' outcomes.

Keywords: lung cancer, multidisciplinary care, clinical pathways

\section{Introduction}

The Institute of Medicine, the American Society of Clinical Oncology, and the European Society of Medical Oncology have promoted delivery of multimodality treatment by a multidisciplinary team of skilled health providers. ${ }^{1,2}$ Multidisciplinary clinics (MDCs) have been associated with enhanced coordination of care, better adherence to clinical guidelines, and improved use of evidence-based care. ${ }^{3}$ Additionally, the incorporation of MDCs has been shown to reduce time from evaluation to start of therapy ${ }^{4}$ and improve patient satisfaction scores. ${ }^{5}$ The Association of Community Cancer Centers published guidelines for the provision of multidisciplinary care in the community, recommending that "an organized, consistent multidisciplinary team approach to the care of patients with cancer and their families is necessary to ensure that needs are identified, interventions are planned, treatments are coordinated, and care is monitored and evaluated."6 A recent publication described the efforts of National Cancer Institute-designated community cancer centers ${ }^{7}$ to develop multidisciplinary care in their respective institutions 
and provided a blueprint of necessary building blocks in the development of these clinics. ${ }^{8}$

Stage III non-small-cell lung cancer (NSCLC) defines a heterogeneous group of diseases. Determining appropriate therapies for this diverse group of patients underscores the need for a coordinated approach to treatment, involving surgery, medical oncology, radiation oncology, pulmonary medicine, navigation, and other ancillary services. This careful coordination of treatment is best facilitated through an MDC. Adequate staging is essential in making sound treatment recommendations, and the coordination of all three therapeutic disciplines must be established to ensure best outcomes. ${ }^{9}$ Coordinated care decreases redundancy of tests and improves compliance with clinical pathways. Expediency of care improves patient's satisfaction and may impact survival.

More than 400 new lung cancer cases are seen annually at Lehigh Valley Health Network (LVHN), which has supported a thoracic MDC since 2007. Fifteen percent of patients with NSCLC at our institution presented with stage III disease. Approximately two-thirds of patients with stage III NSCLC are seen initially in the outpatient setting; the remaining onethird are diagnosed at the time of hospitalization. The patients identified in the outpatient setting are either referred directly to the thoracic MDC or to an individual physician outside of the MDC setting. We compare patients with stage III NSCLC seen in the thoracic MDC and patients with stage III NSCLC not seen in the MDC. This analysis will underscore the need for a multidisciplinary approach to the treatment of patients with stage III lung cancer.

\section{Methods}

\section{Sample}

All patients with stage III NSCLC (American Joint Committee on Cancer 7 [AJCC]) seen at LVHN between March 2010 and March 2013 were identified from tumor registry data and were included in the analysis. The histologic diagnosis of NSCLC and stage was confirmed by one of the physicians participating in the thoracic MDC.

\section{Structure of the MDC}

The thoracic MDC at LVHN meets weekly for prospective case reviews. Physicians from thoracic surgery, medical oncology, radiation oncology, diagnostic radiology, and pulmonary medicine attend the clinic. Representatives from palliative medicine and nutrition also attend each clinic. Prior to each meeting, a nurse navigator reviews all cases and collects pertinent clinical data, including a full history, radiology studies, and pathology (both reviewed internally) for presentation to the group. After group discussion, and if additional testing is necessary, the nurse navigator will schedule tests before treatment recommendations are made. If no additional testing is needed, the team formulates a coordinated treatment plan, which is presented to the patient and family at the time of the initial visit. The patient and family meet all members of the team when the treatment plan is presented, then meet individually with treating physicians to discuss details of therapy. If additional referrals are needed (smoking cessation, pulmonary rehabilitation, and pain management), they are made by the nurse navigator.

\section{Data collection and analysis}

Demographic data were collected for all patients. The dates of each patient's radiographic studies, biopsy, initial visit with a treating physician, and start of therapy were recorded. In addition, if either endobronchial ultrasound (EBUS) or mediastinoscopy was performed, the date of the procedure was documented.

A patient was designated as an "MDC patient" if he or she was referred directly to the thoracic MDC for treatment. Conversely, a patient was designated a "non-MDC patient" if the patient was referred to a treating physician's (medical oncology, radiation oncology, and thoracic surgery) primary office and seen separately by the treating physicians. If seen outside of the MDC, all treating physicians who saw the patients prior to initiation of therapy (and dates of visits) were identified.

The following time intervals were calculated: 1) time from initial radiographic studies to biopsy (bronchoscopy or computed tomography [CT] guided), 2) time from initial radiographic studies to "first touch" by a treating physician or MDC, 3) time from initial radiographic studies to initiation of therapy, and 4) time from the first touch by a treating physician or MDC to initiation of treatment. We also examined adherence to institutional clinical pathways and out-migration of patients from the LVHN system. Overall survival data of patients seen in the MDC were compared to those patients seen outside of the MDC. Finally, patient satisfaction surveys were sent to all patients seen in the thoracic MDC.

This study was approved by the Lehigh Valley Health Network Institutional Review Board.

\section{Statistical analysis}

Continuous variables are presented as mean \pm standard deviation (SD) or median (25th to 75 th percentile). The independent Student's $t$-test was used to assess statistical significance of difference between the groups presented as mean \pm SD. If the distributions were highly skewed, Mann-Whitney $U$ 
test was utilized to test for differences, and medians and percentiles are presented.

Categorical variables are presented as frequency (percentage). These variables were tested using the chi-squared test, with the exception of out-migration, which was tested using Fisher's exact test. All tests of significance were two tailed, and $P$-values of $<0.05$ were considered significant.

Kaplan-Meier survival curves and the log-rank test are used to test survival between those seen in the MDC and patients who are not.

\section{Results}

Two hundred and twenty patients with stage III NSCLC were identified in the tumor registry between March 2010 and March 2013. Sixty-four patients were excluded from the analysis for the reasons outlined in Table 1. An additional 47 patients with proven stage III NSCLC were seen initially by treating physicians during an in-hospital admission. These patients therefore did not fulfill the criteria of MDC or nonMDC and were also excluded from the analysis. There were 52 patients identified as MDC patients and 57 patients identified as non-MDC patients. Over the 3-year period, an increasing percentage of patients with stage III NSCLC was initially seen in the MDC as opposed to outside it: in 2010, 13 patients (34.2\%) were seen in the MDC; in 2011, 21 patients $(53.8 \%)$; and in 2012, 18 patients $(56.3 \%) ; P=0.116$.

Age, sex, and stage were similar between the two groups of patients. More patients with squamous cell carcinoma were seen in the MDC cohort (24 [46.2\%]) than in the nonMDC cohort (14 [24.6\%]) $(P=0.026)$. The higher percentage of patients with squamous cell histology seen in the MDC cannot be explained. Patients' referral to either the MDC or the non-MDC setting by their primary care provider (PCP) was dependent upon the PCP's referral patterns. Patients were not assigned to MDC or non-MDC on the basis of histology.

Table I Number of patients with stage III NSCLC identified in the tumor registry but excluded from analysis and reasons for exclusion

\begin{tabular}{ll}
\hline Reason for exclusion & $\begin{array}{l}\text { Number of } \\
\text { patients }\end{array}$ \\
\hline Small cell lung cancer & 9 \\
Pleural or pericardial metastases or effusion & 35 \\
(stage IV by AJCC 7) & \\
Distant metastatic disease & 12 \\
Other histology & 4 \\
Lesser stage & 4 \\
\hline
\end{tabular}

Abbreviations: NSCLC, non-small-cell lung cancer; AJCC, American Joint Committee on Cancer.

\section{Non-MDC visits}

Fifty-seven patients were initially seen outside of the MDC setting. None of these patients were sent to the MDC for evaluation prior to initial treatment. Twenty-seven patients were initially seen in the thoracic surgery practice, 21 underwent surgical resection (wedge, lobectomy, bilobectomy, or pneumonectomy) as their initial therapy. Seven had preoperative, pathological staging of the mediastinum. Of the 21 patients treated with surgical resection, 14 were subsequently evaluated by medical oncology for adjuvant therapy.

Twenty-four patients were initially seen by medical oncology. Four of these patients were referred to thoracic surgery for evaluation, and four were referred directly to hospice. Sixteen patients received either single modality chemotherapy or combined chemoradiation.

Of six patients initially seen by radiation oncology, four received radiation as primary therapy, and two received combined chemoradiation.

\section{Time intervals to treatment}

There was no difference in the median time between initial radiographic discovery of the lung cancer and start of therapy between MDC patients (61 days [51.5-81.5]) and non-MDC patients (54 days [36.0-103.0]) $(P=0.549)$. Similarly, there was no difference in the time from bronchoscopy to treatment in the two cohorts (MDC, 35 days [29.25-54.0]; non-MDC, 33 days [20.25-59.0]) $(P=0.495)$. However, patients seen in the MDC started therapy within a mean of $19.85 \pm 13.8$ days as opposed to those not seen in the MDC, who started therapy at an average of 29.09 \pm 27.3 days $(P=0.043)$. These data are displayed in Figure 1.

\section{Staging of the mediastinum}

When enlarged lymph nodes were present on CT or positron emission tomography (PET)-CT, patients seen in the MDC were more likely to undergo pathological staging of the mediastinum with either mediastinoscopy or EBUS examination. As shown in Figure 2, 30 MDC patients $(57.7 \%)$ had pathological staging of mediastinum with either EBUS (21 patients [40.4\%]) or mediastinoscopy (nine patients [17.3\%]); 14 non-MDC patients $(24.5 \%)$ had pathological staging of the mediastinum with EBUS (six patients [10.5\%]) or mediasinoscopy (eight patients [14\%]) $(P<0.001)$. Of the patients not seen in the MDC, $27(47 \%)$ were seen initially by a thoracic surgeon and were taken to surgery prior to consultation with either medical or radiation oncology. Twenty-one non-MDC patients (36\%) who were taken directly to surgery had N2 involvement discovered at 


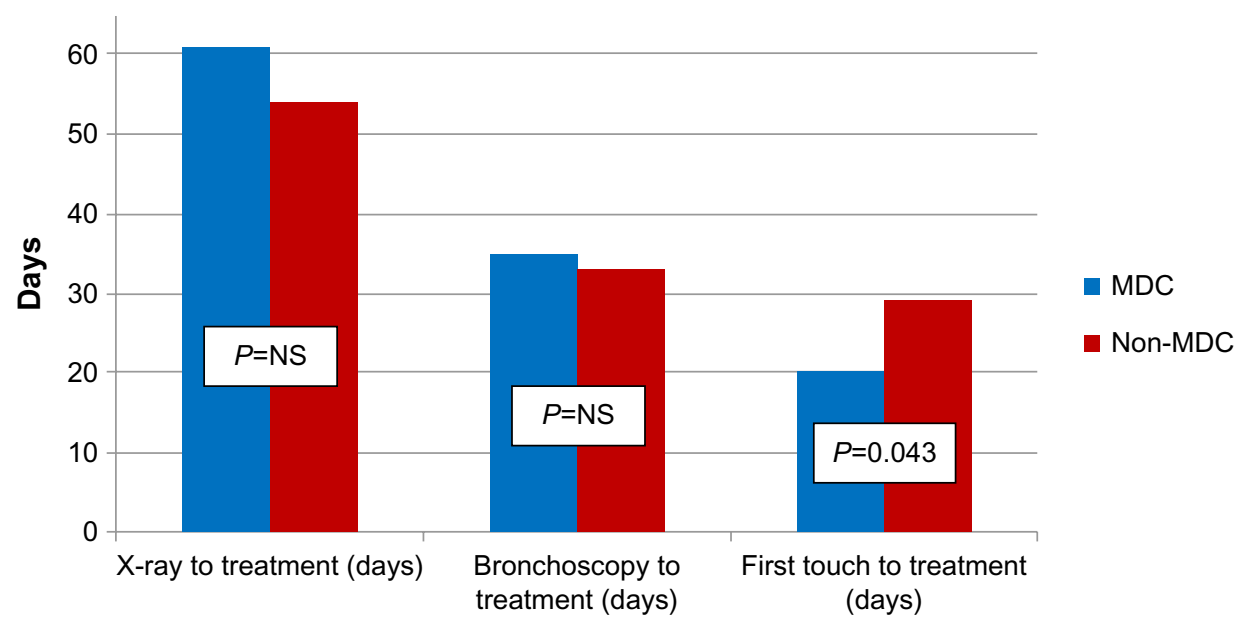

Figure I Time intervals to initiation of treatment.

Abbreviations: MDC, multidisciplinary clinic; NS, not statistically significant.

the time of surgery. Only four MDC patients (7.7\%) treated with surgery initially were found to have N2 lymph node involvement at the time of surgery $(P<0.001)$.

\section{Clinical pathway adherence}

The thoracic malignancy disease management team at LVHN advocates combined modality chemotherapy and radiation therapy either as definitive therapy or in the neoadjuvant setting depending on stage, nodal status, performance status, and comorbid conditions limiting surgery. The development of our lung cancer clinical pathway incorporated these recommendations and is the standard of care at our institution. As shown in Figure 2, 46 patients (88.5\%) seen in the MDC were treated according to the LVHN clinical pathway for stage III
NSCLC as opposed to only 20 patients (35.1\%) seen outside of the MDC $(P<0.001)$.

\section{Overall survival}

Overall survival of patients seen in the MDC was compared with those seen outside of the MDC. Median overall survival for patient seen outside of the MDC was 14 months. Median overall survival for patients seen in the MDC was 17 months $(P=0.054)$. The survival data are displayed in Figure 3 .

\section{Team approach to treatment}

Patients not seen in the MDC may be seen by all or some of the treating physicians prior to initiation of therapy. While 52 patients $(100 \%)$ seen in the MDC were seen by more

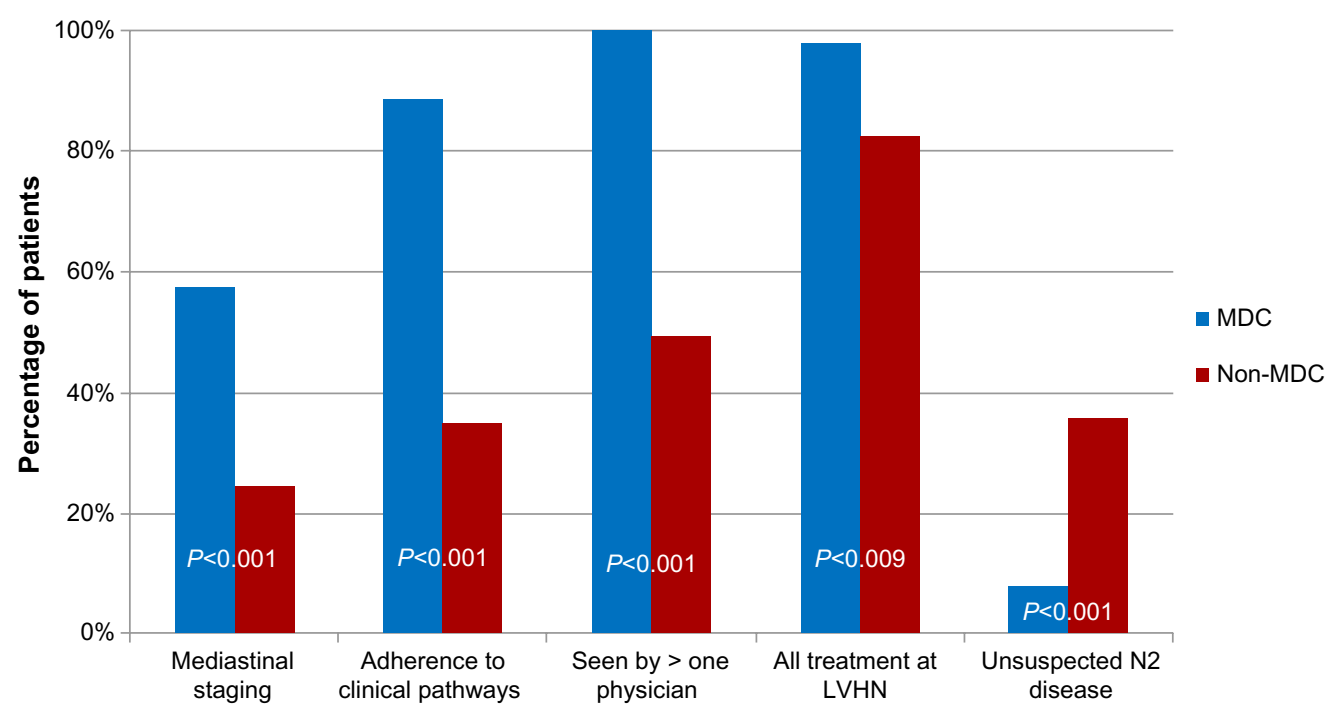

Figure 2 Comparison of MDC and non-MDC patients in clinical treatment planning. Abbreviations: MDC, multidisciplinary clinic; LVHN, Lehigh Valley Health Network. 


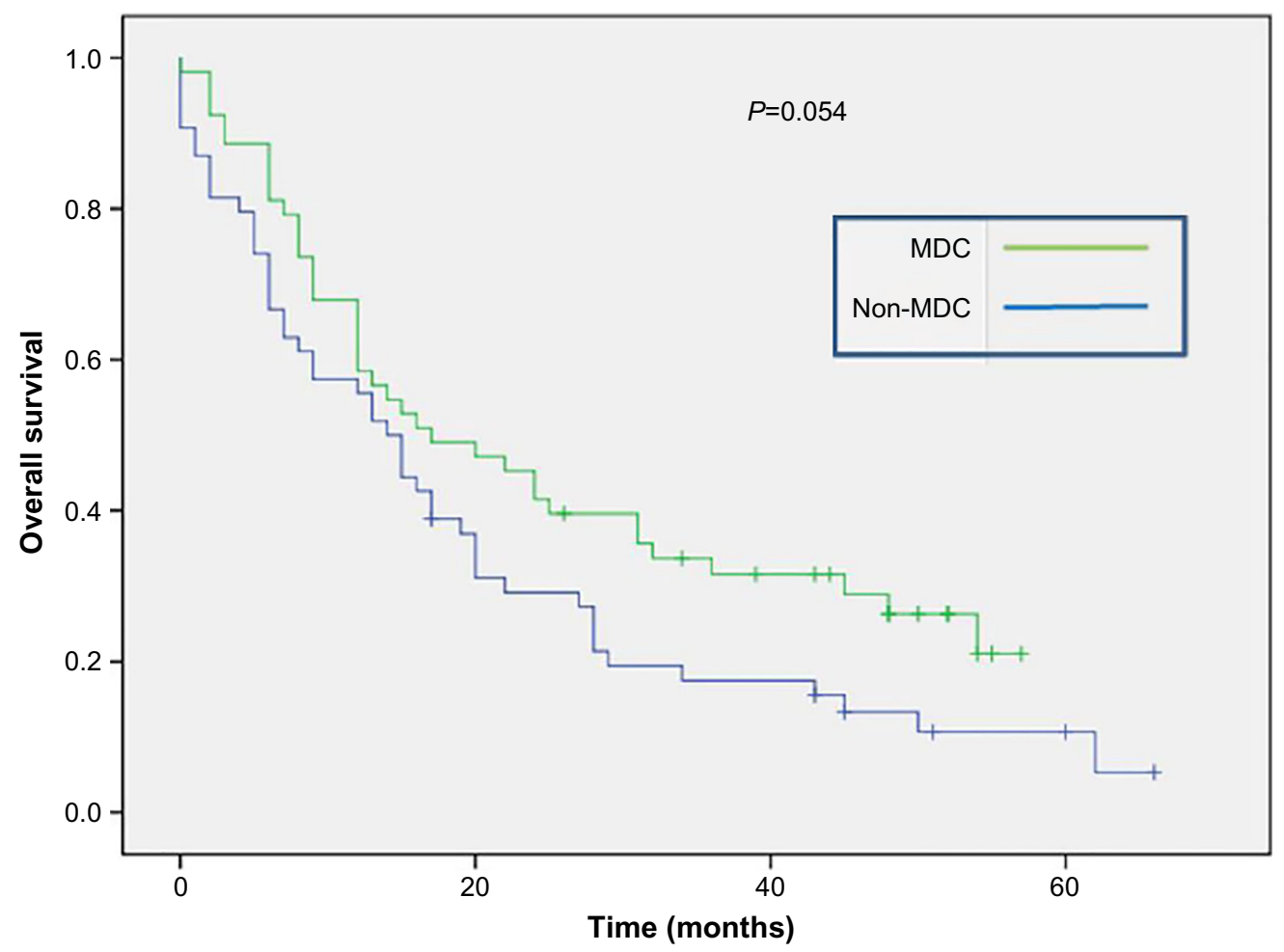

Figure 3 Comparison of overall survival - MDC vs non-MDC patients. Abbreviation: MDC, multidisciplinary clinic.

than one treating physician, 28 patients (49.1\%) not seen in the MDC were seen by more than one physician prior to starting treatment $(P<0.001)$. These data are displayed in Figure 2.

\section{Out-migration from LVHN system}

When initially seen in the thoracic MDC, 51 patients (98.1\%) received their treatment at $\mathrm{LVHN}$, as opposed to 47 patients $(82.5 \%)$ who received therapy at LVHN when not seen in the MDC $(P=0.009)$. These data are displayed in Figure 2.

\section{Patient's satisfaction}

Patients participating in the thoracic MDC were given satisfaction surveys following their visit. Five questions relating directly to physician care were analyzed. The questions were presented as a five-point Likert scale ranging from 1 to 5 : very poor (1), poor (2), fair (3), good (4), and very good (5). There was a $45.4 \%$ response to the survey. The five questions, analyzed with the percentages of "good" and "very good" answers, are demonstrated subsequently.

1. Did the doctor show respect for your questions? (good $-17.8 \%$; very good $-82.2 \%$ )

2. How clearly did the doctor explain your condition and treatment?

(good $-15.4 \%$; very good $-82.9 \%$ )
3. Were you satisfied with the length of time that you spent with the doctor?

(good $-29.3 \%$; very good $-69 \%$ )

4. Were the written recommendations that you received useful?

(good $-26 \%$; very good $-72 \%$ )

5. What is the likelihood of recommending this service to others?

(good $-18.3 \%$; very good $-80.9 \%$ )

Patient satisfaction surveys are also conducted within the individual practices of the LVHN; however, these are anonymous surveys. Moreover, data on patients with thoracic malignancies cannot be separated from the conglomerate of data recorded. Therefore, no clear comparison of MDC and non-MDC patients can be reported.

\section{Insurance comparison of MDC and non- MDC patients}

There was no difference in insurance distribution between patients seen in the MDC and those seen outside of the MDC. Fifty-eight percent of patients seen in the MDC had Medicare as their primary insurance, either alone or with a secondary insurance (including Medicaid). Fifty-one percent of the patients seen outside of the MDC had Medicare with or without a secondary insurance plan ( $P=$ nonsignificant $)$. 


\section{Discussion}

As cancer care becomes more complex, optimal treatment of patients will involve the integrated approach of surgery, radiation therapy, and chemotherapy. The treatment of stage III NSCLC exemplifies the importance of a coordinated treatment approach. Multidisciplinary evaluation of these patients is recommended by the American College of Chest Physicians and the National Cancer Center Network guidelines. ${ }^{10}$ Additionally, the lack of consensus that surrounds therapy of these patients underscores the need to develop more consistent care within institutions. The provision of consistent care and creation of clinical pathways can best be accomplished through coordinated multidisciplinary care and clinics.

Surgical staging of the mediastinum is critical in determining appropriate therapy for patients with stage III NSCLC. EBUS or mediastinoscopy are the gold standards with regard to staging of the mediastinum. CT scan with intravenous contrast or PET-CT may yield either false negative or false positive result. N2 lymph nodes not suspected on CT scan or PET-CT can be detected either by EBUS or mediastinoscopy. The identification of involved N2 or N3 lymph nodes previously not suspected may change the management of these patients. If $\mathrm{N} 2$ adenopathy is identified by either mediastinoscopy or EBUS, chemotherapy as single modality ${ }^{11-14}$ or combined with radiation therapy ${ }^{15-17}$ would be appropriate as either neoadjuvant therapy or definitive treatment. ${ }^{18}$ Furthermore, if N3 adenopathy unsuspected on CT or PET-CT scan was discovered, therapy would involve concomitant chemotherapy with radiation in the definitive mode.

Adherence to clinical pathways and clinical practice guidelines has been shown to ensure quality of care and improve consistency of care in patients with a variety of malignancies. ${ }^{19}$ Without a consensus "gold standard" for the treatment of stage III NSCLC, the management of these patients may differ from institution to institution. These preferences may reflect the resources and experience available at a particular institution. Once institutions develop their paradigm for treatment, care becomes more consistent, and valuable data with regard to outcomes and cost can be collected. These treatment paradigms will ultimately be adopted as the clinical pathway at that cancer center for the treatment of that patient's malignancy.

The thoracic MDC at our institution has adopted concomitant chemotherapy plus radiation as initial treatment for all patients with stage III NSCLC. If patients meet certain treatment criteria, this bimodality therapy will be utilized in the neoadjuvant setting. ${ }^{20}$ However, if they do not meet criteria for surgery, combined chemotherapy and radiation therapy will be used as definitive treatment. This multimodality approach and complex decision making requires coordinated care among thoracic surgeons, medical and radiation oncologists, and pulmonologists. The MDC ensures that patients are seen by physicians from each modality prior to initiation of treatment. When patients are not seen in the clinic, this coordinated approach to care cannot be guaranteed.

The benefit of institutional clinical pathways, accurate staging of the mediastinum, and a combined team approach to care of these complex patients is underscored by an improvement in median overall survival in our MDC patients compared to non-MDC patients in results that approach significance. We believe that consistency of care, input from all treating physicians, and a unified approach to therapy will continue to yield improved outcomes in this group of patients.

In many institutions, physicians are held accountable for relative value unit generation and revenue production. As a result, individual physicians who do not see patients in an MDC setting may treat these patients by utilizing their own treatment modality and not confer with colleagues before starting treatment. This practice is exemplified by the fact that, at LVHN, institutional guidelines were followed $\sim 90 \%$ of the time when seen in the MDC. When patients were not seen in the MDC, guidelines were followed $<50 \%$ of the time. ${ }^{21}$ Although there are always exceptions that preclude treating all patients according to clinical pathways, it is generally accepted that adherence to these pathways should occur at least $80 \%$ of the time.

In today's complex medical milieu, many hospital systems and networks are developing accountable care organizations in accordance with the Medicare Shared Savings Program. ${ }^{22,23}$ When patients are not managed in a single system, the cost of medical care may increase and the lag time to begin therapy increases as well. It is common for oncology patients seeking second opinions at major cancer centers to undergo additional radiologic tests and review of pathology obtained elsewhere. Among radiologists, there is an inherent mistrust of studies not done at their own institution and subsequent unwillingness to comment on scans done elsewhere. This redundancy causes increased fees, unnecessary radiation exposure, and an overall boost in the cost of care. Conversely, cost of care may be reduced when patients are treated at a single site. ${ }^{24}$

The multidisciplinary model may increase patient confidence in the original institution. LVHN is situated within 
1.5 hours of three world-renowned cancer centers. If a patient sees a provider at LVHN and then goes for a second opinion at a neighboring cancer center, the reputation of the other cancer center could influence the patient's decision to be treated elsewhere. Conversely, in the MDC setting, a patient is seen simultaneously by several physicians, has the ability to ask questions regarding his or her care in a controlled environment, and is presented with a unified care plan. As a result, the patient's satisfaction with the MDC is high and the likelihood that the patient will seek a second opinion or treatment elsewhere may be diminished. We believe that the retention rate of patients seen in our thoracic MDC relative to the retention rate of those not seen in the MDC supports the fact that patients have increased confidence in LVHN when seen in the MDC. Additionally, they are receiving care at a single institution, potentially impacting the cost of their treatment and engendering high patient's satisfaction. As we have demonstrated, patients seen in the MDC setting experience more immediate initiation of care, which potentially reduces the chance that the cancer will progress and, thus, not be treated as effectively.

Future efforts at our institution will focus on decreasing the time from the initial detection of the malignancy to bronchoscopy and from bronchoscopy to treatment. Additionally, PCPs are being encouraged to send their patients directly to the thoracic MDC when an abnormality on a chest X-ray or CT scan is detected. An earlier referral will allow a more expedient determination of pathological diagnosis, ensure appropriate staging, and expedite the start of treatment. Finally, the palliative medicine service at LVHN actively participates in the thoracic MDC. All patients with stage IV NSCLC are referred to this service when seen either in the clinic or in the medical oncology office. We are beginning to evaluate the utility of palliative medicine in earlier stages of NSCLC, especially during combined treatment with chemotherapy and radiation, in which there may be toxicity.

\section{Conclusion}

This study provides insights into the value of a thoracic MDC in the treatment of stage III NSCLC. Our data illustrate overall quantitative improvements in several aspects of care for these patients. As we move forward with our MDC and add patients to this study, we will be able to conduct more detailed subgroup analysis and gain additional information regarding the value of the MDC in caring for patients with stage III NSCLC. If institutions have the available resources, the multidisciplinary setting should be used for all patients with this stage of disease. We believe that a coordinated treatment approach, treatment in accordance with established guidelines, and prospective planning will ultimately show an economic advantage and improved clinical outcomes.

\section{Acknowledgment}

The authors are grateful to Jacqueline Grove for assistance with manuscript preparation.

\section{Disclosure}

The authors report no conflicts of interest in this work.

\section{References}

1. Levit BL, Nass S, Ganz PA, editors. Delivering High-Quality Cancer Care: Charting a New Course for a System in Crisis. Washington, DC: National Academies Press; 2013.

2. American Society of Clinical Oncology. European Society for Medical Oncology. ASCO-ESMO consensus statement on quality cancer care. Ann Oncol. 2006;17(7):1063-1064.

3. Tripathy D. Multidisciplinary care for breast cancer: barriers and solutions. Breast J. 2003;9(1):60-63.

4. Frost MH, Arvizu RD, Jayakumar S, Schoonover A, Novotny P, Zahasky K. A multidisciplinary healthcare delivery model for women with breast cancer: patient satisfaction and physical and psychosocial adjustment. Oncol Nurs Forum. 1999;26(10):1673-1680.

5. Horvath LE, Yordan E, Malhotra D, et al. Multidisciplinary care in the oncology setting: historical perspective and data from lung and gynecology multidisciplinary clinics. J Oncol Pract. 2010;6(6):e21-e26.

6. Association of Community Cancer Centers (ACCC) [webpage on the Internet]. Clinical Management and Supportive Care Services - the Multidisciplinary Team. Cancer Program Guidelines. 2009. Available from: http://www.accc-cancer.org/publications/CancerProgramGuidelines-4.asp. Accessed February 9, 2016.

7. Johnson MR, Clauser SB, Beveridge JM, O’Brien DM. Translating scientific advances into the community setting: the National Cancer Institute Community Cancer Centers program pilot. Oncol Issues. 2009;24(3):24-28.

8. Friedman EL, Chawla N, Morris PT, et al. Assessing the development of multidisciplinary care: experience of the National Cancer Institute Community Cancer Centers Program. J Oncol Pract. 2015;1: e36-e43.

9. de Cabanyes CS, Detterbeck FC. A systematic review of restaging after induction therapy for stage IIIa lung cancer: prediction of pathologic stage. J Thorac Oncol. 2010;5(3):389-398.

10. Ramnath N, Dilling TJ, Harris LJ, et al. Treatment of stage III non-small cell lung cancer: diagnosis and management of lung cancer, 3rd edition: American College of Chest Physicians evidence-based clinical practice guidelines. Chest. 2013;143(5 suppl):e314S-e340S.

11. Roth JA, Fossella F, Komaki R, et al. A randomized trial comparing perioperative chemotherapy and surgery with surgery alone in resectable stage IIIA non-small-cell lung cancer. $J$ Natl Cancer Inst. 1994;86(9):673-680.

12. Decaluwé H, De Leyn P, Vansteenkiste J, et al. Surgical multimodality treatment for baseline resectable stage IIIA-N2 non-small cell lung cancer. Degree of mediastinal lymph node involvement and impact on survival. Eur J Cardiothorac Surg. 2009;36(3):433-439.

13. Garrido P, González-Larriba JL, Insa A, et al. Long-term survival associated with complete resection after induction chemotherapy in stage IIIA (N2) and IIIB (T4N0-1) non small-cell lung cancer patients: the Spanish Lung Cancer Group Trial 9901. J Clin Oncol. 2007;25(30):4736-4742.

14. Stefani A, Alifano M, Bobbio A, et al. Which patients should be operated on after induction chemotherapy for N2 non-small cell lung cancer? Analysis of a 7-year experience in 175 patients. $J$ Thorac Cardiovasc Surg. 2010;140(2):356-363. 
15. Yokomise H, Gotoh M, Okamoto T, et al. Induction chemoradiotherapy (carboplatin-taxane and concurrent 60 Gy radiation) for bulky cN2, N3 non-small cell lung cancer. J Thorac Cardiovasc Surg. 2007;133(5):1179-1185.

16. Friedel G, Budach W, Dippon J, et al. Phase II Trial of a trimodality regimen for stage III non-small-cell lung cancer using chemotherapy as induction treatment with concurrent hyperfractionated chemoradiation with carboplatin and paclitaxel followed by subsequent resection: a single-center study. J Clin Oncol. 2010;28(6):942-948.

17. Suntharalingam M, Paulus R, Edelman MJ, et al. Radiation therapy oncology group protocol 02-29: a phase II trial of neoadjuvant therapy with concurrent chemotherapy and full-dose radiation therapy followed by surgical resection and consolidative therapy for locally advanced non-small cell carcinoma of the lung. Int J Radiat Oncol Biol Phys. 2012;84(2):456-463.

18. Albain KS, Crowley JJ, Turrisi AT 3rd, et al. Concurrent cisplatin, etoposide, and chest radiotherapy in pathologic stage IIIB non-smallcell lung cancer: a Southwest Oncology Group phase II study, SWOG 9019. J Clin Oncol. 2002;20(16):3454-3460.
19. Smith TJ, Hillner BE. Ensuring quality cancer care by the use of clinical practice guidelines and critical pathways. J Clin Oncol. 2001;19(11): 2886-2897.

20. Friedman E, Szwerc M, Andrews C, Kruklitis R, Leies K, Santiago F. Neo-adjuvant chemotherapy and radiation therapy in patients with stage IIIA (N2) non-small cell lung cancer (NSCLC): a retrospective analysis with lessons learned. Poster presented at: The 14th World Conference on Lung Cancer; July 2011, Amsterdam, The Netherlands.

21. Casey DE Jr. Why don't physicians (and patients) consistently follow clinical practice guidelines? JAMA Intern Med. 2013;173(17): $1581-1583$.

22. Lee TH, Casalino LP, Fisher ES, Wilensky GR. Creating accountable care organizations. $N$ Engl J Med. 2010;363(15):e23.

23. Greaney TL. Accountable care organizations - the fork in the road. N Engl J Med. 2011;364(1):e1.

24. Neubauer MA, Hoverman JR, Kolodziej M, et al. Cost effectiveness of evidence-based treatment guidelines in the treatment of non-small-cell lung cancer in the community setting. J Oncol Pract. 2010;6(1):12-18.
Journal of Multidisciplinary Healthcare

\section{Publish your work in this journal}

The Journal of Multidisciplinary Healthcare is an international, peerreviewed open-access journal that aims to represent and publish research in healthcare areas delivered by practitioners of different disciplines. This includes studies and reviews conducted by multidisciplinary teams as well as research which evaluates the results or conduct of such teams or health-

\section{Dovepress}

care processes in general. The journal covers a wide range of areas and welcomes submissions from practitioners at all levels, from all over the world. The manuscript management system is completely online and includes a very quick and fair peer-review system. Visit http://www.dovepress.com/ testimonials.php to read real quotes from published authors. 